The submitted manuscript has been authored by a contractor of the U.S. Govemment under Contract No. DE-AC05-96OR22464. Accordingly, the U.S. Government retains a nonexclusive royalty-free license to publish or reproduce the published form of this contribution, or allow others to do so, for U.S. Govemment purposes.

\title{
Numerical Tokamak Turbulence Calculations on the CRAY T3E
}

\author{
V. E. Lynch, J-N. Leboeuf, B. A. Carreras \\ Oak Ridge National Laboratory, Oak Ridge, TN 37831 \\ J. D. Alvarez, L. Garcia \\ Universidad Carlos III de Madrid, Spain

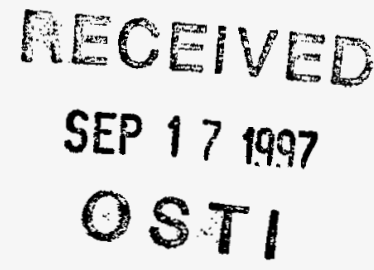

\begin{abstract}
Full cross section calculations of ion-temperature-gradient-driven turbulence with Landau closure are being carried out as part of the Numerical Tokamak Turbulence Project, one of the U. S. Department of Energy's Phase II Grand Challenges. To include the full cross section of a magnetic fusion device like the tokamak requires more memory and CPU time than is available on the National Energy Research Scientific Computing Center's (NERSC's) shared-memory vector machines such as the CRAY C90 and J90. Calculations of cylindrical multihelicity ion-temperature-gradient-driven turbulence were completed on NERSC's 160-processor distributed-memory CRAY T3E parallel computer with 256 Mbytes of memory per processor. This augurs well for yet more memory and CPU intensive calculations on the next-generation T3E at NERSC. This paper presents results on benchmarks with the current T3E at NERSC. Physics results pertaining to plasma confinement at the core of tokamaks subject to ion-temperature-gradient-driventurbulence are also highlighted. Results at this resolution covering this extent of physical time were previously unattainable. Work is in progress to increase the resolution, improve the performance of the parallel code, and include toroidal geometry in these calculations in anticipation of the imminent arrival of a fully configured, 512-processor, T3E-900 model.
\end{abstract}

* Research sponsored by the Office of Fusion Energy, U.S. Department of Energy, under contract DE-AC05-96OR22464 with Lockheed Martin Energy Research Corp.

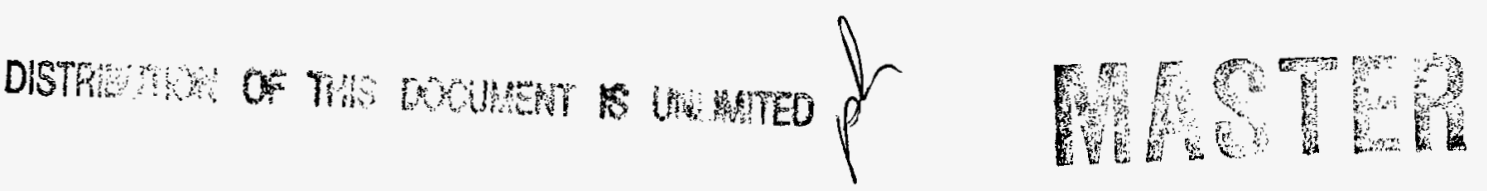




\section{DISCLAIMER}

This report was prepared as an account of work sponsored by an agency of the United States Government. Neither the United States Government nor any agency thereof, nor any of their employees, make any warranty, express or implied, or assumes any legal liability or responsibility for the accuracy, completeness, or usefulness of any information, apparatus, product, or process disclosed, or represents that its use would not infringe privately owned rights. Reference herein to any specific commercial product, process, or service by trade name, trademark, manufacturer, or otherwise does not necessarily constitute or imply its endorsement, recommendation, or favoring by the United States Government or any agency thereof. The views and opinions of authors expressed herein do not necessarily state or reflect those of the United States Government or any agency thereof. 


\section{Introduction}

Anomalous ion transport in tokamaks seems to be consistent with ion-temperaturegradient-driven turbulence. Present theoretical transport models based on this type of turbulence give a good description of present data, but the quantitative agreement is not yet good enough for transport predictions on future devices [1]. To further develop these models, a better understanding is needed of the turbulence dynamics, particularly near marginal stability.

The underlying linear instability of ion-temperature-gradient-driven turbulence is the so-called $\eta_{i}$ mode [2] (where $\eta_{i}$ is the ratio of the density to the ion temperature scale lengths, $\left.\eta_{i} \equiv\left[d \ln \left(T_{i}\right) / d r\right] /\left[d \ln \left(n_{i}\right) / d r\right]\right)$. This instability is essentially an electrostatic sound wave that is driven unstable by the ion pressure gradient. This instability is triggered by $\eta_{i}$ becoming greater than a threshold value, $\eta_{i t h}$. The $\eta_{i}$ instability has a variety of branches in different regimes [3]. The first analytical results for the derivation of an ion thermal conductivity, $\chi_{i}$, based on this instability were derived for $\eta_{i}$ well above the stability threshold, where a fluid approximation could be applied $[4,5]$. The value for $\chi_{i}$ obtained in these calculations was too high when compared with the experiment. The fact that $\eta_{i}$ instabilities can lead to such high levels of losses suggests that a marginal stability condition could constrain the profiles [6]. The determination of the marginal stability criterion and the linear eigenmode close to the threshold requires the use of kinetic theory and full toroidal geometry. Therefore, the complexity of the problem has increasingly demanded the use of numerical calculations.

A new opportunity emerged with the development of gyro-fluid models with Landau closure $[7,8]$. The possibility of including Landau damping (at least in the linear approximation) in fluid models has extended the computational capabilities for studying ion-temperature-gradient-driven turbulence $[9,10]$. These techniques led to a new approach for calculating the anomalous diffusivities [11]. However, present nonlinear calculations are limited by the use of flux-tube calculation domains. This limitation is particularly important because of the role that global shear flows play on the saturation of the turbulence. Since the global shear flow is the outcome of Reynolds stress flow amplification, flow boundary conditions can determine the results. It is also not clear what role the limited extent of these flux tubes plays in determining the topology of the flows.

Here, full torus fluid calculations of ion-temperature-gradient-driven turbulence with Landau closure have been initiated for eventual comparison with flux tube models of ion-temperature-gradient-driven turbulence. To keep these calculations at a computationally manageable level, we only include time evolution equations for the perturbed ion density (vorticity) and parallel velocity, in addition to a perturbed ion temperature equation in which a simple parallel linear Landau closure is imposed. In this simplified model, the electrons are treated as adiabatic. Finite differences in radius and Fourier mode expansions in poloidal and toroidal angles allow us to describe the full geometry. This permits the exploration of the tractability of nonlinear calculations covering the full plasma cross section compared to a radially localized toroidal annulus.

The issue of full toroidal calculations becomes an issue of problem size for a given level of resolution. Because the characteristic length scale is the ion Larmor radius, $\rho_{i}$, to resolve this scale in a fluid calculation, we need about 10 grid points per $\rho_{\mathrm{i}}$. For a mediumsize experiment, the plasma radius is about $200 \rho_{\mathrm{i}}$; therefore, about 2000 radial grid points are needed. Resolving similar poloidal scales requires poloidal mode number values of about 200 , and the number of Fourier components needed is about 15000 . This level of numerical resolution implies a memory size requirement that is about an order of magnitude above the capabilities of the present T3E at NERSC. However, this resolution could be reached in the next generation T3E. We can also reduce the size of the problem to fit in the 
present computer by reducing the radial extent of the calculation. This is what is done in this paper, where we study the optimal conditions for a reduced problem and present the result of the calculation.

The rest of this paper is organized as follows. The set of equations used to simulate the ion-temperature-gradient-driven turbulence is presented in Sect. 2, together with the numerical discretization scheme. The implementation of this scheme on a multiple-CPU system is described in Sect. 3, where we also discuss the optimization studies for calculations done on the T3E at NERSC. The results of single- and multiple-helicity calculations are presented in Sect. 4. In Sect. 5 we give the main conclusions of this study. 


\section{Equations and Numerical Scheme}

In this study, we consider the simplest form of an ion-temperature-gradient-driven turbulence model that incorporates Landau closure. The plasma geometry is taken to be cylindrical, with $r$ being the radial coordinate and $\theta$ the poloidal angle. The model is derived using the perpendicular ion momentum balance to extract the perpendicular ion velocity, and, from the ion continuity equation, to obtain an evolution equation for the electrostatic potential, $\tilde{\Phi}$,

$$
\begin{aligned}
\frac{\partial}{\partial t} \nabla_{\perp}^{2} \tilde{\Phi} & -\frac{|e| B_{0}^{2}}{n_{e q} M c^{2}} \frac{\partial \tilde{n}}{\partial t}=\frac{|e| B_{0}^{2}}{n_{e q} M c^{2}} \nabla_{\|} \tilde{V}_{\| i}-\frac{|e| B_{0}}{n_{e q} M c} \frac{d n_{e q}}{d r} \frac{1}{r} \frac{\partial \tilde{\Phi}}{\partial \theta}, \\
& -\frac{c T_{i}^{e q}}{|e| B_{0} n_{e q}} \frac{d n_{e q}}{d r}\left(1+\eta_{i}\right) \frac{1}{r} \frac{\partial}{\partial \theta}\left(\nabla_{\perp}^{2} \tilde{\Phi}\right)-\frac{c}{B_{0}}[(\hat{z} \times \nabla \tilde{\Phi}) \cdot \nabla] \nabla_{\perp}^{2} \tilde{\Phi},
\end{aligned}
$$

where, $M$ is the ion mass, $|e|$ is the ion charge, and $c$ is the speed of light. From the parallel ion momentum balance, an equation for the evolution of the parallel velocity is derived,

$$
\frac{\partial}{\partial t} \tilde{V}_{\| i}=-\frac{1}{M} \nabla_{\|} \tilde{T}_{i}-\frac{T_{i}^{e q}}{n^{e q} M} \nabla_{\|} \tilde{n}-\frac{|e|}{M} \nabla_{\|} \tilde{\Phi}-\frac{c}{B_{0}}[(\hat{z} \times \nabla \tilde{\Phi}) \cdot \nabla] \tilde{V}_{\| i}
$$

Finally, the ion temperature evolution equation is

$$
\frac{\partial \tilde{T}_{i}}{\partial t}=-T_{i}^{e q} \nabla_{\|} \tilde{V}_{\| i}+\frac{c}{B_{0}} \frac{d T_{i}^{e q}}{d r} \frac{1}{r} \frac{\partial \tilde{\Phi}}{\partial \theta}-\left[\frac{2^{2 / 3}}{\sqrt{\pi}}\left|k_{\|}\right| V_{T_{i}}\right] \tilde{T}_{i}-\frac{c}{B_{0}}[(\hat{z} \times \nabla \tilde{\Phi}) \cdot \nabla] \tilde{T}_{i} .
$$

The last linear term of Eq. (3) is the Landau damping term. In this model, electrons are assumed to be adiabatic, $\tilde{n} / n_{e q}=|e| \tilde{\Phi} / T_{e}^{e q}$. This condition closes the system of equations. In these equations the index $e q$ indicates equilibrium quantities and the tilde refers to fluctuating quantities.

The time-advanced variables in Eqs. (1) to (3) are the ion temperature, $T_{\mathrm{i}}$, the ion parallel velocity, $V_{\| l}$, and the $z$-component of the vorticity, $\nabla_{\perp}^{2} \tilde{\Phi}$. From the latter, the potential and density fluctuations are derived. The equilibrium electron temperature, $T_{e}^{e q}$, and density, $n_{e q}$, are supposed to be constant in time. Only the ion temperature and poloidal-velocity-averaged profiles are modified by the turbulence. The total magnetic field can be expressed in terms of the equilibrium poloidal flux function, $\Psi_{0}$, as $B=-\left(\nabla \Psi_{0} \times \hat{z}\right) / R_{0}+B_{0} \hat{z}$, where $B_{0}$ is the toroidal component of the magnetic field. The derivative parallel to the magnetic field, $\nabla_{\mathrm{ll}}$, is defined as $\nabla_{\mathrm{ll}} f=B \cdot \nabla f$.

Equations (1) to (3) are solved by using finite differences in the radial coordinate $r$ and Fourier series expansion in the angle variables $\theta$ and $\zeta$ [12]. Equilibrium quantities are functions of the radius only. The fluctuating quantities have the following representation: 


$$
\tilde{f}(r, \theta, \zeta)=\sum_{n=0}^{\infty} \sum_{m=0}^{\infty}\left[f_{m, n}^{c}(r)(\cos m \theta+n \zeta)+f_{m, n}^{s}(r)(\sin m \theta+n \zeta)\right]
$$

In mode number space, only a narrow wedge of $m$ 's and $n$ 's are resonant in the plasma $[q(r)=m / n$ where $q$ is the safety factor and $m$ and $n$ denote poloidal and toroidal mode numbers, respectively]. The radial extent of the calculation restricts the range of $q$ values and, therefore, the distribution of Fourier components and size of the radial region are strongly coupled.
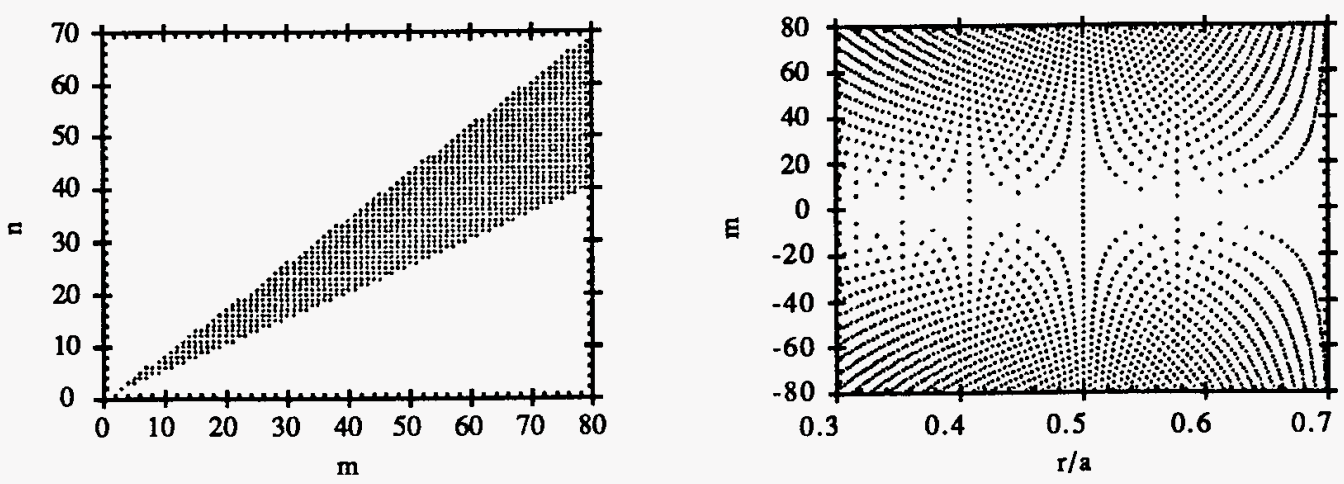

Fig. 1. Distribution of Fourier components included in the calculation.

Details of the numerical discretization of the equations can be found in ref. [12] After the Fourier expansion, Eqs. (1) to (3), the time evolution equations can symbolically be represented as

$$
L_{m, n} \frac{\partial X_{m, n}}{\partial t}=R_{m, n} X_{m, n}+N_{m, n}(X),
$$

where, $\mathrm{X}$ is a vector whose components are the Fourier components of the three timeadvanced variables. The numerical scheme is time-implicit for linear terms, the first term on the right hand side of Eq. (5), and is time-explicit for nonlinear terms (N). These nonlinear terms are quadratic nonlinearities which become convolutions of the Fourier components. In the numerical calculation, these convolutions are treated analytically. To numerically advance these equations, a two-step, second-order-accurate, time-centered advancement scheme is used.

$$
\begin{aligned}
& L_{m, n} X_{m, n}^{i+1 / 2}=\left(L_{m, n}+\frac{\Delta t}{2} R_{m, n}\right) X_{m, n}^{i}+\frac{\Delta t}{2} N_{m, n}\left(X^{i}\right), \\
& \left(L_{m, n}-\frac{\Delta t}{2} R_{m, n}\right) X_{m, n}^{i+1}=\left(L_{m, n}+\frac{\Delta t}{2} R_{m, n}\right) X_{m, n}^{i}+\Delta t N_{m, n}\left(X^{i+1 / 2}\right) .
\end{aligned}
$$

For this numerical scheme, the main computational tasks are the inversion of the matrices $L$ [for Eq. (6)] and $L-\Delta t R / 2$ [for Eq. (7)] and the calculation of the convolutions twice for each time step. 


\section{Multi-CPU Implementation of the Numerical Scheme}

Using the time advancement scheme in Eqs. (6) and (7), most of the floating point operations are in the convolutions in the nonlinear terms and in the matrix operations for the solution of the time-implicit linear terms. The convolutions are done analytically. Fast Fourier transforms are not used because only a narrow wedge of $m$ 's and $n$ 's are resonant in the plasma. The convolutions are done in complex exponential form and are banded in $m$ and $n$ space. The matrix inversion is accomplished by a block-tridiagonal matrix decomposition done at the beginning of the calculation and then solving the linear system twice in each time step.

PVM is used for the multiprocessor implementation of this scheme on the T3E. The serial code is replicated on all processors used. Only matrix operations for the timeimplicit linear terms and convolutions for the time-explicit nonlinear part of the calculation are distributed to multiple processors. For matrix operations, parallelization is done for all radial grid points at once over the number of Fourier harmonics in which all physical quantities in the problem are expanded. For the convolutions, parallelization is done over the number of radial grid points for all Fourier harmonics at once. Memory for the matrices is allocated at run time and depends on the number of processors requested for the calculation. Since memory is relocated in going from matrix inversion to the convolutions and vice versa, a global send and receive is done using PVM after all the linear matrix solutions and after all the convolutions in each time step.

Flow traces of the time spent in called routines have been generated. Figure 2 shows the time distribution among the different types of operations for a calculation with 424 grid points and 2351 Fourier components run on 16 processors. Two versions of the banded convolution routine have been written. One is for a vector machine with vectorization over the radial dimension. The other is for cache-based machines with the outer loop over the radial dimension for maximum re-use of cache residency. The vector routine executes faster than the cache-based one on the T3E. Work is underway to understand why this routine is faster and to optimize the coding.

There are two communication subroutines in this code. One follows the calculation of the convolutions and data is packed in many short arrays, one per radial slice. The second follows the matrix inversion and the same amount of data is packed in fewer, but longer, arrays, one per Fourier component. Each way of packing is a consequence of the way data is stored in each processor at that time. The first subroutine takes much more time than the second. To improve efficiency, a second version of the communication subroutine following the convolutions was implemented. In this version, the packing routine was called with longer arrays, of the length of the number of Fourier components instead of the number of grid points per processor. With the communications handled in this way, the percentage of time spent in the communication routine for the convolutions decreases by about a factor of 2, as shown in Fig. 3. The linear solve routines and their communication routine are not as time-consuming, by more than a factor of 20 compared to the convolutions. The replicated serial coding used primarily for setup at the beginning of the calculation takes an insignificant percentage of the time. 


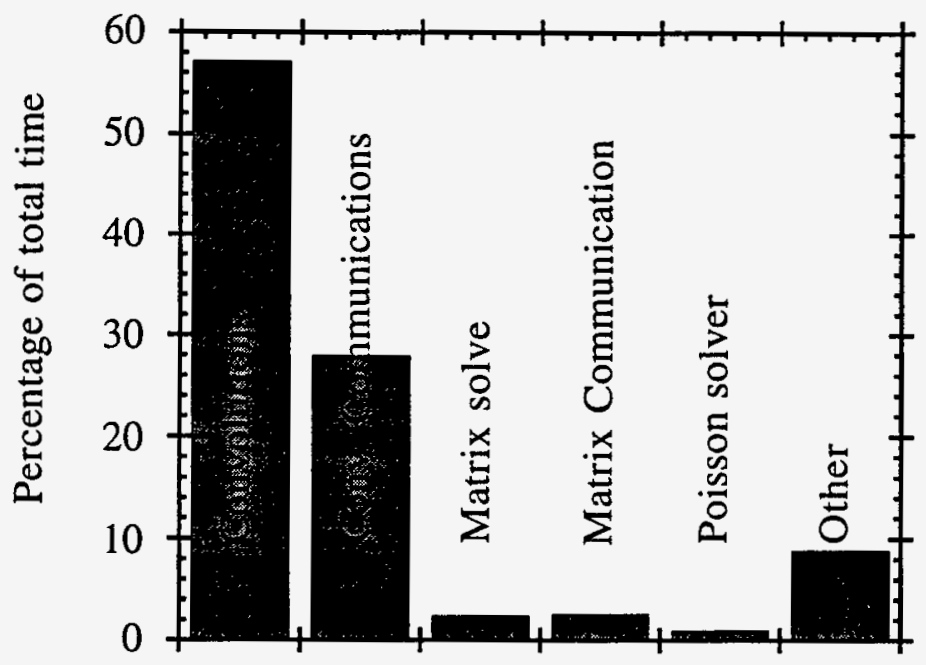

Fig. 2. Time distribution among the different types of operations for a calculation run on 16 processors before optimization of the convolution communications.

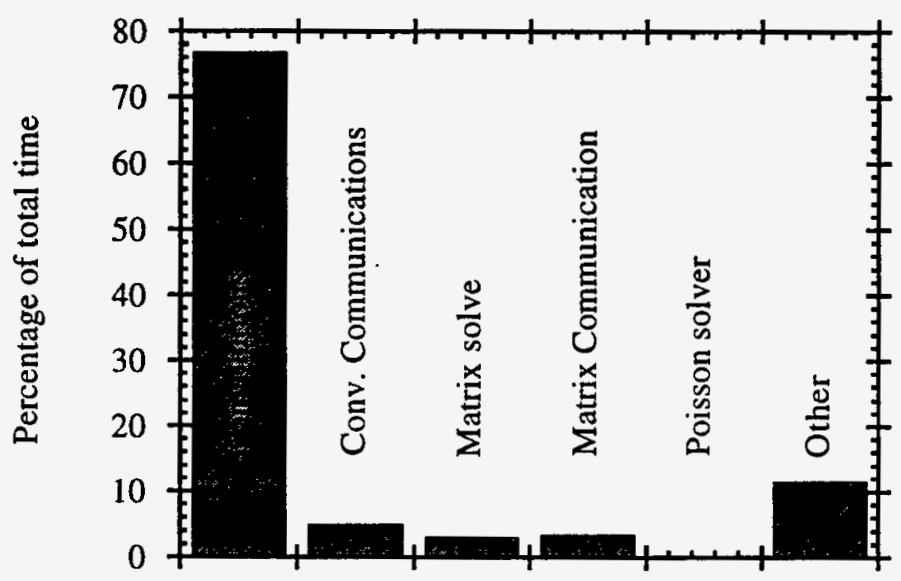

Fig. 3. Time distribution among the different types of operations for a calculation run on 16 processors after optimization of the convolution communications.

Here we consider two different problem sizes: one uses the maximum capacity of the Cray Y-MP/C90 and another uses the maximum capacity of the present T3E. The first allows us to compare the efficiency of both machines, the second to test the capabilities of the T3E. The latter calculation is still an order of magnitude below the resolution requirements for a full-size plasma experiment.

The smaller nonlinear calculation was carried out with 559 Fourier components and with the radial extent restricted by the small number of resonant modes, represented with 
420 grid points. It has been performed on both the Cray Y-MP/C90 [13] and the T3E. Identical results were obtained on both machines over 5200 time steps. On the C90 the average number of concurrent processors was between 4 and 5 , while 9 processors were used on the T3E. The elapsed seconds per time step averaged 1.6 for the C90 and 5.37 for the T3E. The shared memory requirements were $30.6 \mathrm{Mword}$ for the $\mathrm{C} 90$ and the memory per processor was 8.3 Mword for the T3E. For the calculation with 559 Fourier components on the T3E, the number of processors, $n p$, was varied from 1 to 64 to calculate the speed-up (time on one processor / time on np processors) as a function of the number of processors. This is the largest calculation that can be run on one processor, since each processor has 32 Mword of memory. The maximum speed-up was 8 for 32 processors (Fig. 4). Since the efficiency decreased as we went beyond nine processors, nine processors were used.

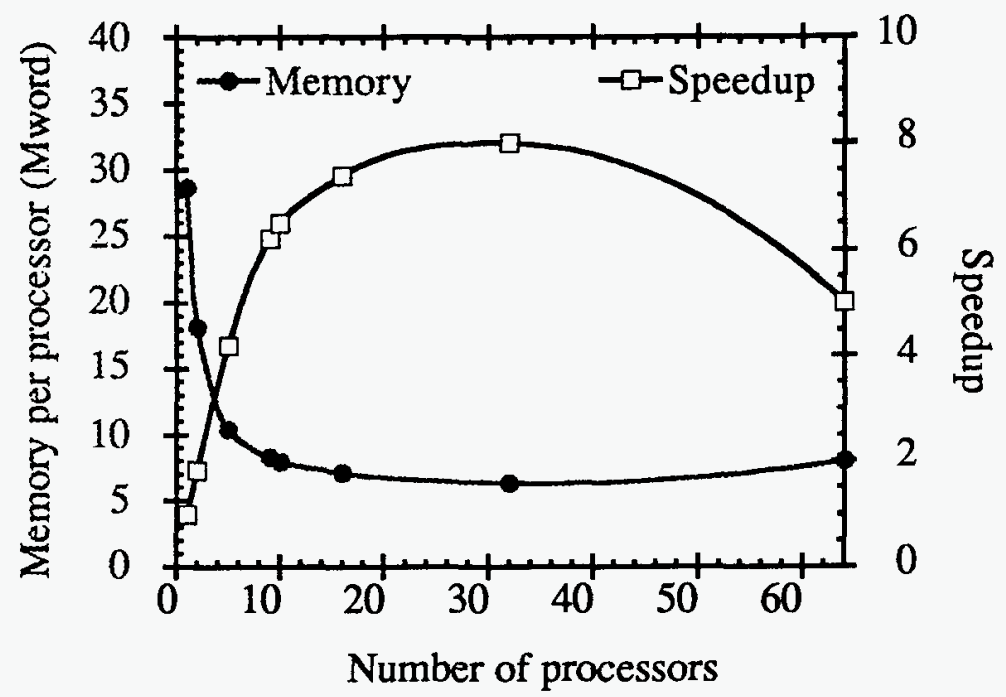

Fig. 4. Memory used per processor and speed-up factor as a function of number of processors for a calculation using 559 Fourier components and 420 radial grid points.

The larger calculation was done for 2351 Fourier components and 424 grid points. This calculation is too large for the C90 and was completed on the T3E. This production calculation was run for 26,850 time steps in many short batch runs (because of batch queue time limitations) over a few weeks. Physics and restart data were stored every 50 time steps. A partial analysis of the resulting 12 GBytes worth of information is presented in Sect. 4. In order to calculate the optimal number of processors, the number of T3E processors was varied to see its effect on the elapsed time per step. We used the same criterion for optimal performance as in ref. 12: that CPU time per step be reduced less than $25 \%$ as the number of processors is increased by a factor of 2 . For the first version of the code, in which short arrays are used in the PVM packing routine, the optimal number of processors was 32 . After the PVM routine was called with fewer but longer arrays, the optimal number of processors was increased to 90 and the elapsed time for each step was reduced by a factor of 2 as shown in Fig. 5. With this optimization in the convolution communications, calculations with more grid points and more Fourier components over a larger number of processors are now possible. To date, a performance of 2.2 Gflops has been achieved with 128 processors. It should be noted that in this case $42 \%$ of the elapsed time is spent in loading instructions and data caches. We further remark that the loading and 
cache time increases linearly with the number of processors and at the same rate as the communications time.

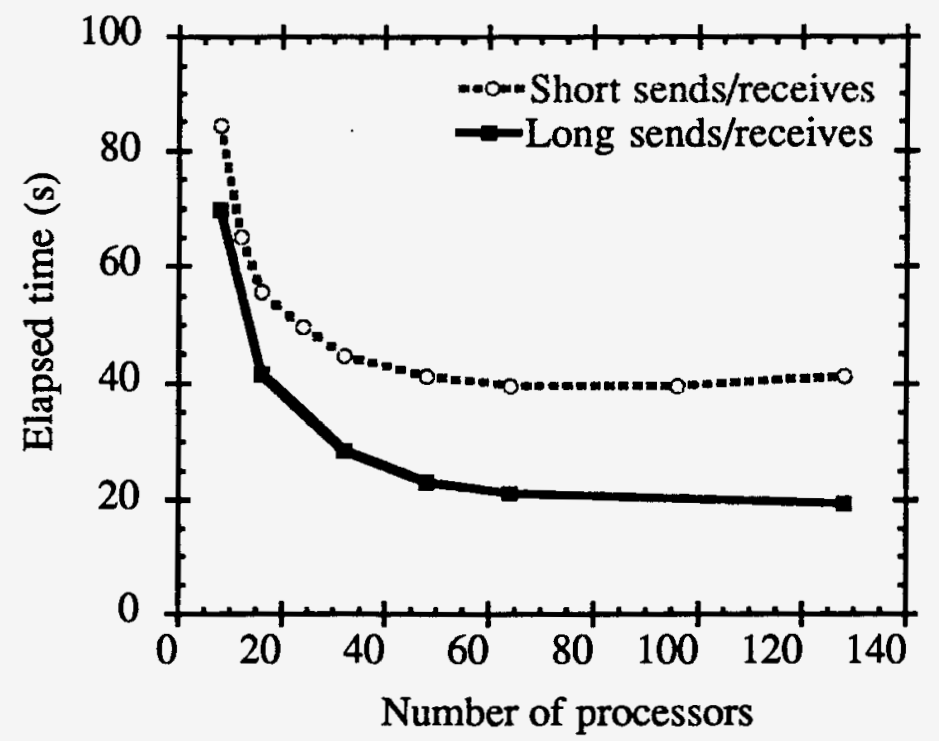

Fig. 5. Reduction of the calculation time with increasing number of processors for calculations before and after optimization of convolution communication.

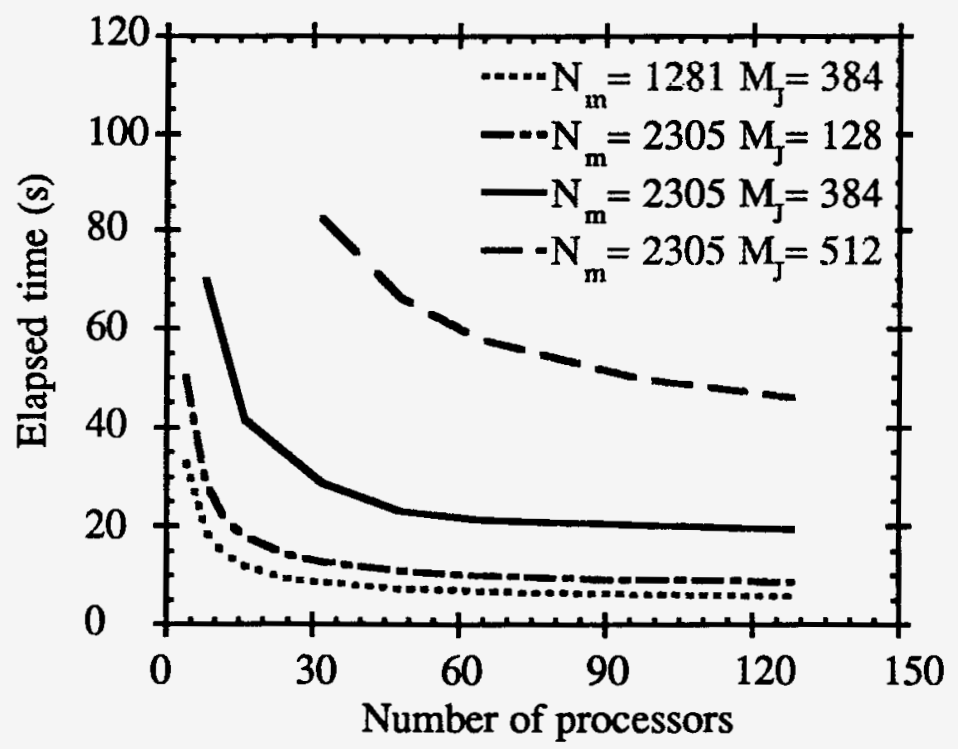

Fig. 6. Reduction in calculation time with an increasing number of processors for calculations with varying resolution in numbers of Fourier components $\mathrm{N}_{\mathrm{m}}$ and grid points MJ.

The numbers of grid points and Fourier components were also varied to see the effect of the number of T3E processors on the elapsed time per time step. This was done to estimate the number of processors needed for a calculation with increased resolution. The 
optimal number of processors increased from 64 to 90 and up to 128 as the radial grid was increased from 128 to 384 and up to 512 , and from 64 to 90 as the number of Fourier components was increased from 1281 to 2305 , as shown in Fig. 6. Since the convolutions are divided among processors radially and this is the most time-consuming routine, the optimal number of processors shows more of an increase as the radial grid is increased than when the number of Fourier components is increased. The improvement made in the matrix inversion has led to results that are different from those of the iPSC/860 where the matrix solve routines took a significant percentage of the elapsed time per time step [14]. The optimization task now becomes improving the convolution techniques beyond the improvement in communications described here. 


\section{Results of the Nonlinear Calculations}

For the calculations presented in this paper, the plasma geometry is a circular cross section geometry. The plasma minor radius, $a$, is such that $\rho_{\mathrm{i}}(0) / a=1 / 100$ [with $\rho_{\mathrm{i}}(0)$ indicating the Larmor radius at $\mathrm{r}=0$ ] and the assumed aspect ratio $R / a=8.33$. This corresponds to about one-half of the radius of a medium-size tokamak experiment. Since we have restricted the radial range to $(0.3 \leq r / a \leq 0.7)$, the present study is limited to onefourth of our simulation goal.

Existing calculations have shown that the nonlinear evolution of the iontemperature-gradient-driven turbulence leads to averaged plasma profiles that are close to marginal stability to $\eta_{\mathrm{i}}$ modes. This seems also to be true in the case of the experimentally measured density and temperature profiles. For this reason, the nonlinear calculations presented in this paper were carried out for a value of $\eta_{i}$ relatively close to marginal stability. For the $3 / 2$ helicity, resonant at $r / a=0.5$, marginal stability is $\eta_{\mathrm{i}} \approx 0.8$. Therefore, $\eta_{i}=1.2$ was a reasonable compromise, being close to marginal but large enough to have characteristic times which are not too long. The equilibrium $q, n_{\mathrm{eq}}$, and $T_{\mathrm{i}}^{\text {eq }}$ profiles used for these calculations are shown in Fig. 7.

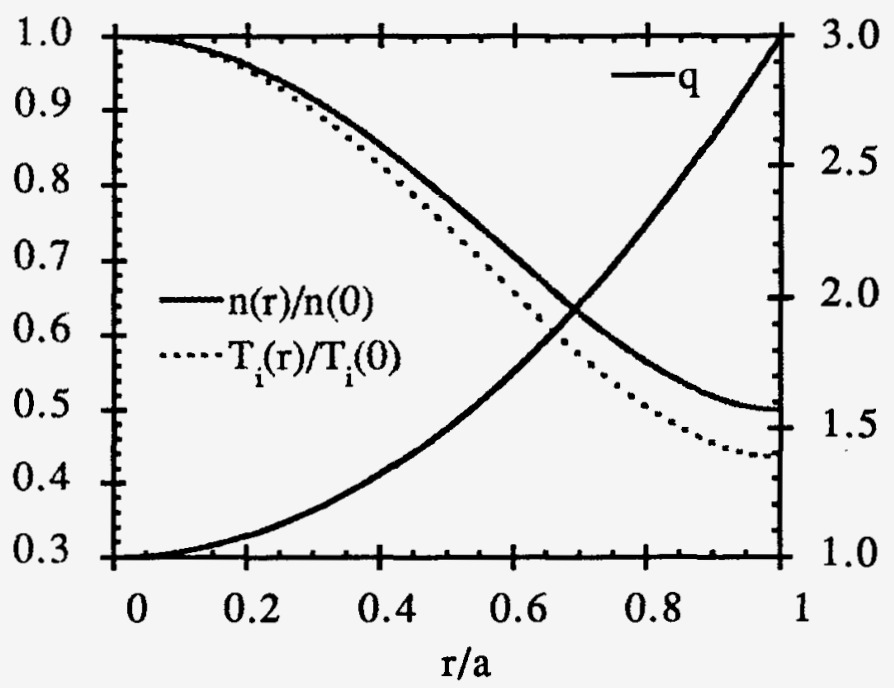

Fig. 7. Equilibrium $q, n_{\text {eq }}$, and $T_{\text {ieq }}$ profiles used in the nonlinear calculations.

The main dimensionless parameters characterizing the properties of the instability are: $L_{\mathrm{n}} / \rho_{\mathrm{i}}$ and $L_{\mathrm{s}} / L_{\mathrm{n}}$, where $L_{\mathrm{n}}$ and $L_{\mathrm{s}}$ are the density and the magnetic shear scale length respectively. For these profiles they vary: $180>L_{\mathrm{n}} / \rho_{\mathrm{i}}>115$ and $55>L_{\mathrm{s}} / L_{\mathrm{n}}>15$. Here we also take the equilibrium ion and electron temperatures to be equal.

The effect of the Landau damping terms in this model was first tested on the linear stability of the $\eta_{i}$ modes. As expected, there is a significant reduction in the linear growth rates with the Landau closure term. This is shown in Fig. 8, where the linear growth rates of the modes in the 3/2 helicity for two values of $\eta_{i}$ are plotted. For each value of $\eta_{i}$, the growth rates are compared with the growth rate obtained in the absence of the Landau term.

Single-helicity calculations were carried out to see the effect of the localization by Landau damping of the instability, and to understand the dynamics of the nonlinear saturation mechanisms. This single-helicity calculation was done with the ITGDT (for ion 
temperature gradient driven turbulence) code, where 109 Fourier components and 420 grid points were used. The fluctuation amplitude grows exponentially in the linear phase and saturates to a relatively low level $(<1 \%)$. The interesting issue is that there is significant localization of the fluctuations with Landau damping on, as opposed to when the Landau damping term is not included. This localization is maintained during the full nonlinear evolution and there is no evidence of outgoing drift waves coupled to the fluctuations (Fig. 9).
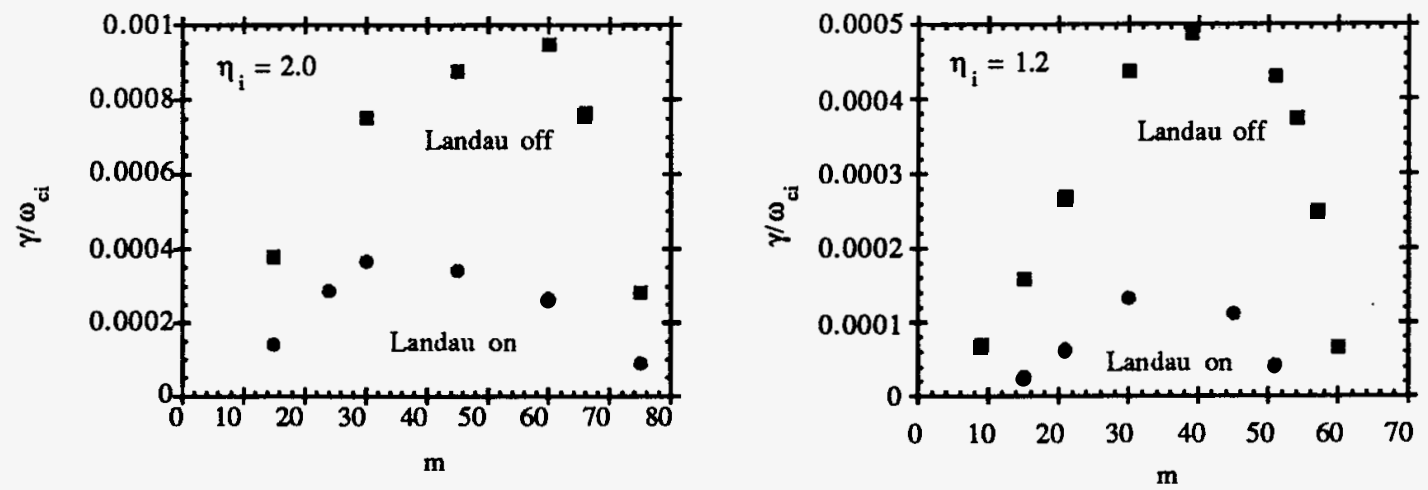

Fig. 8. Linear growth rate with and without Landau damping term for two values of $\eta_{i}$.
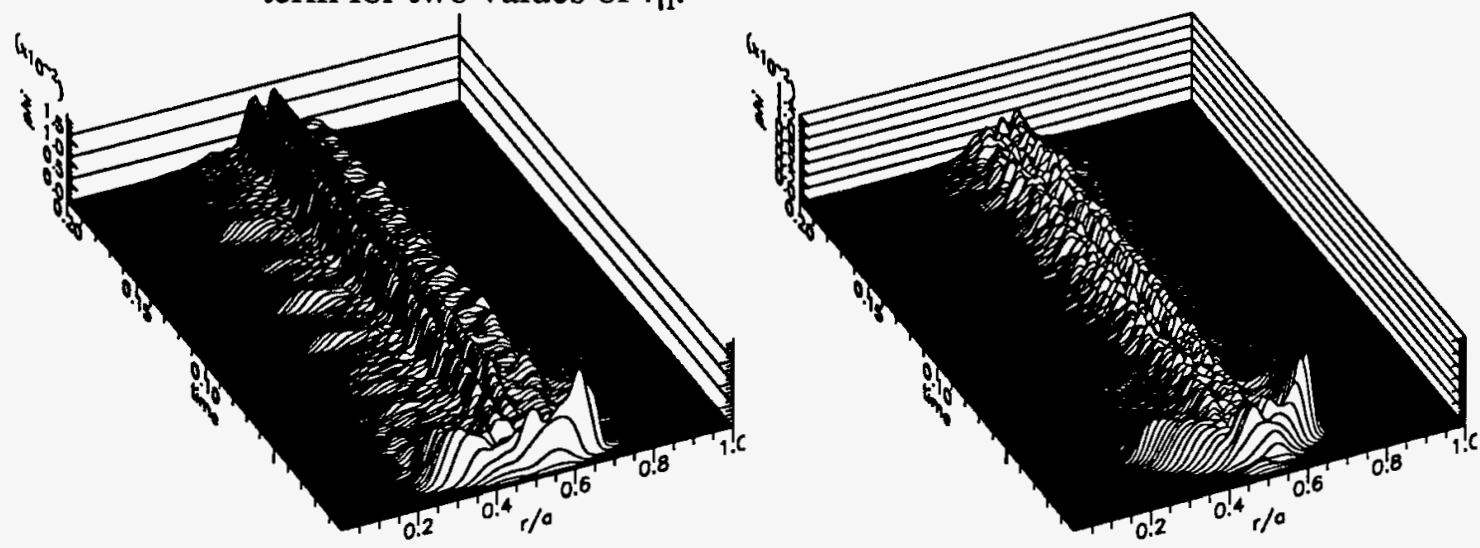

Fig. 9. Nonlinear evolution of the fluctuation profile without (left) and with (right) Landau damping.

The next step in this study is to carry out full 3-D calculations. We have considered cases with different radial extent to vary the size of the problem in trying to understand the efficiency of the algorithm implementation. We have also considered cases with different numbers of nonlinear couplings to understand turbulent saturation mechanisms. The Fourier component distribution is shown in Fig. 1. The calculation was done for $\eta_{i}=1.2$, and with 2351 Fourier components and 424 grid points. The nonlinear evolution showed clear differences from the single-helicity case. In Fig. 10, we have plotted the time history of the fluctuations and the heat flux at the center of the computational box, $r / a=0.5$. The time evolution shows a large overshoot in the initial phase of the nonlinear calculation, followed by a nearly steady state phase. However, there is a slow decay of the fluctuations in this second phase. This is probably caused by the fact that the averaged ion temperature profile has been modified by the fluctuations so that the profile becomes linearly stable to all $\eta_{i}$ modes towards the end of the nonlinear phase. 


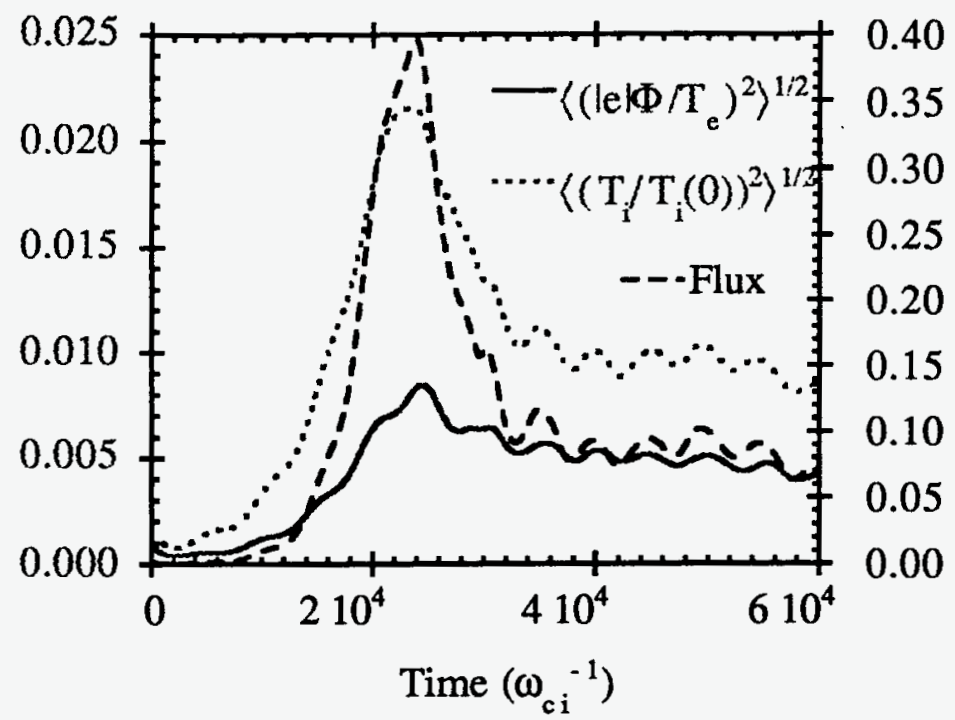

Fig. 10. Time history of the rms fluctuation levels and heat flux at the center of the computational box, $r / a=0.5$.

In the steady state phase of the turbulence, the spectrum of the Fourier components is well converged (Fig. 11). It has a clear peak, and this peak shifts to higher $m$ values as we move towards the plasma edge region. This indicates that the use of a constant $m$ value as spectral cutoff was not the best choice. The cutoff in $m$ has to increase linearly with $r$. The linear stability theory gives $k_{\theta} \rho_{i} \approx\left(1+\eta_{i}\right)^{-1 / 2}$ for the maximum linear growth rate; for the present parameters we have $m_{\max } \approx 67 r / a$. The peak of the turbulence spectrum is close to the maximum of the linear growth rate for the different helicities, as can be seen in Fig. 11.

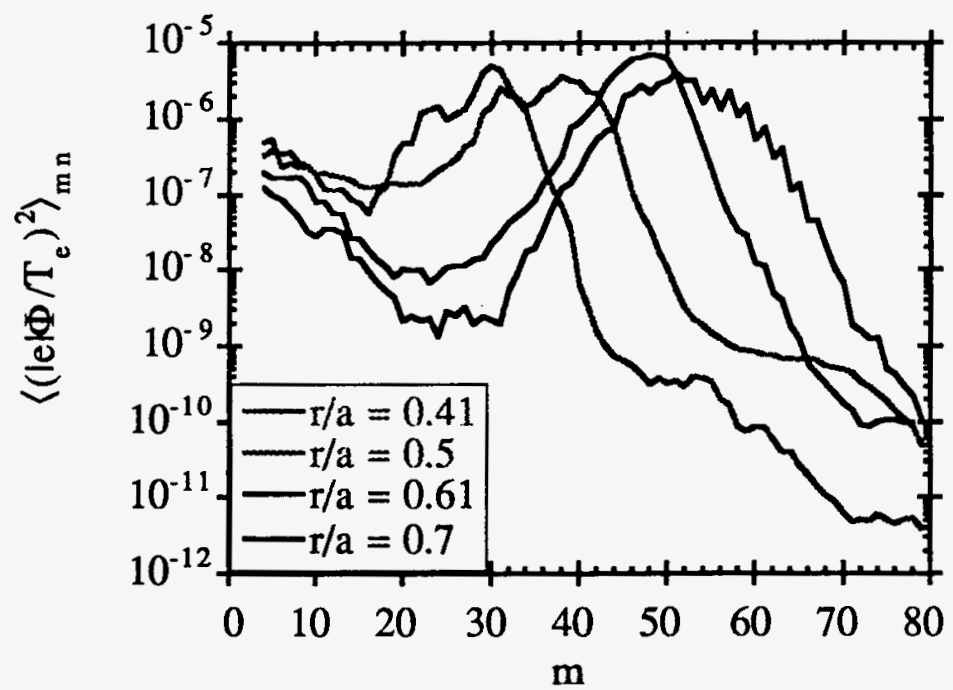

Fig. 11. Spectrum of the Fourier components of the electrostatic potential fluctuations at different radial positions averaged over the steady state phase. 
The radial scales of the turbulence are of the order of $\rho_{\mathrm{i}}$. The radial correlation length in the steady state phase is about $3.0 \rho_{\mathrm{i}}$. No large-scale structures are observed (Fig. 12). This indicates that the induced transport is local and the scaling is gyro-Bohm.

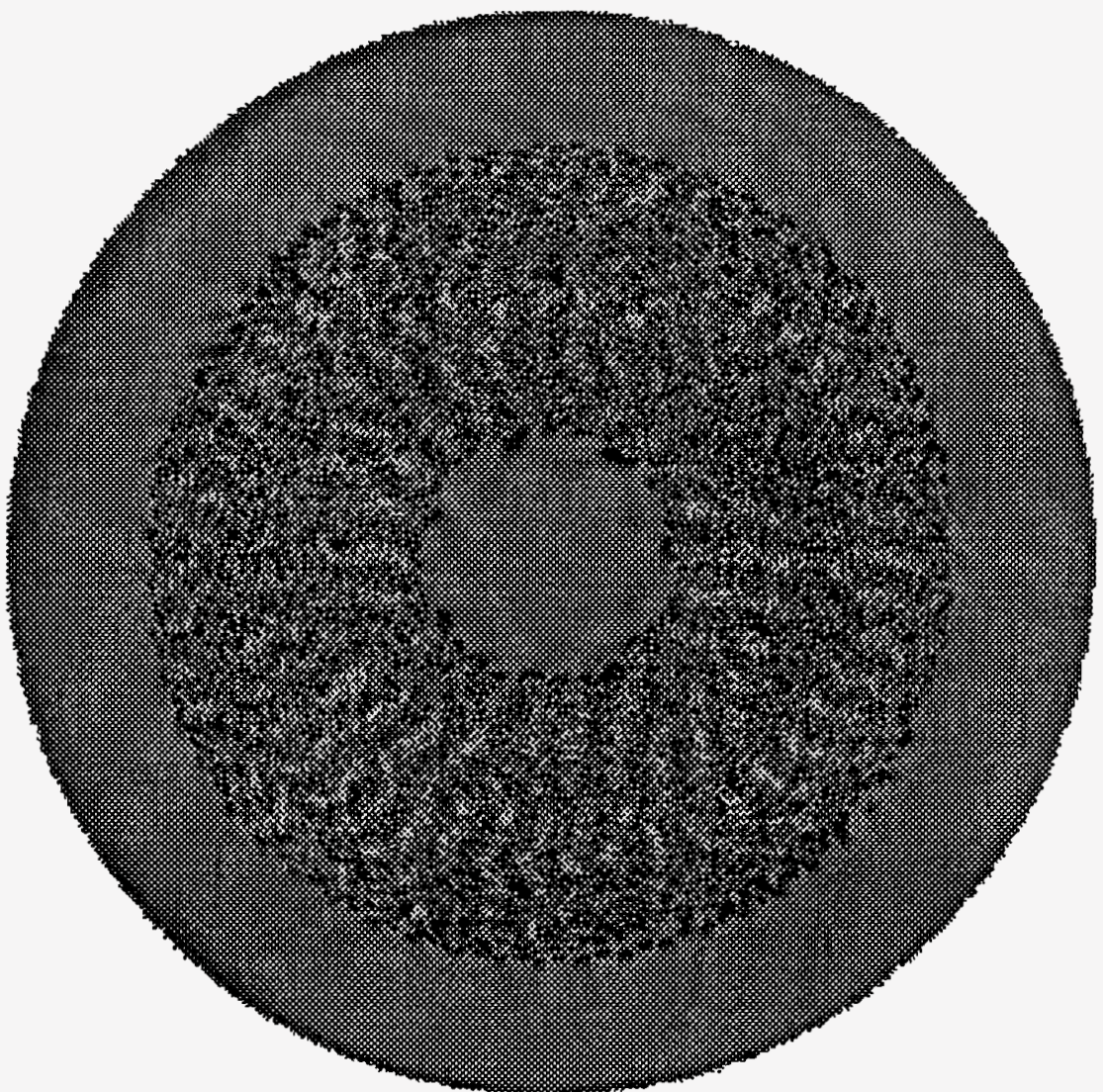

Fig. 12. Contour of constant $T_{\mathrm{i}}$ fluctuations in the steady state of the turbulence.

The turbulence-induced thermal diffusivity, time-averaged over the steady state phase of the calculation, is $\chi_{i} \approx 1.0 \times 10^{-3} \rho_{i}(0) V_{i}(0)$.

The time evolution of the fluctuations shows that they remain radially localized during the whole nonlinear phase (Fig. 13). Fluctuation levels are somewhat larger than in the single-helicity case. This is not surprising because of the larger free energy source (broad radial range) and the cross couplings between helicities. A quasi-periodic oscillation in the fluctuation level is observed during the steady state of the turbulence.

In the nonlinear regime, a relatively large averaged poloidal flow is generated through Reynolds stress. The largest flow is at the transition regions (inside and outside) where the number of modes decreases and there is a large radial inhomogeneity of the radial fluctuation profile (Fig. 14). This effect raises the question of the proper treatment of the sheared poloidal flow in a flux tube. In this case, the radial inhomogeneity is given by the flux tube boundaries that are artificially set up. Inside the radial region where the calculation is performed, the fluctuation level is effectively flat and the sheared poloidal flow is negligible. 


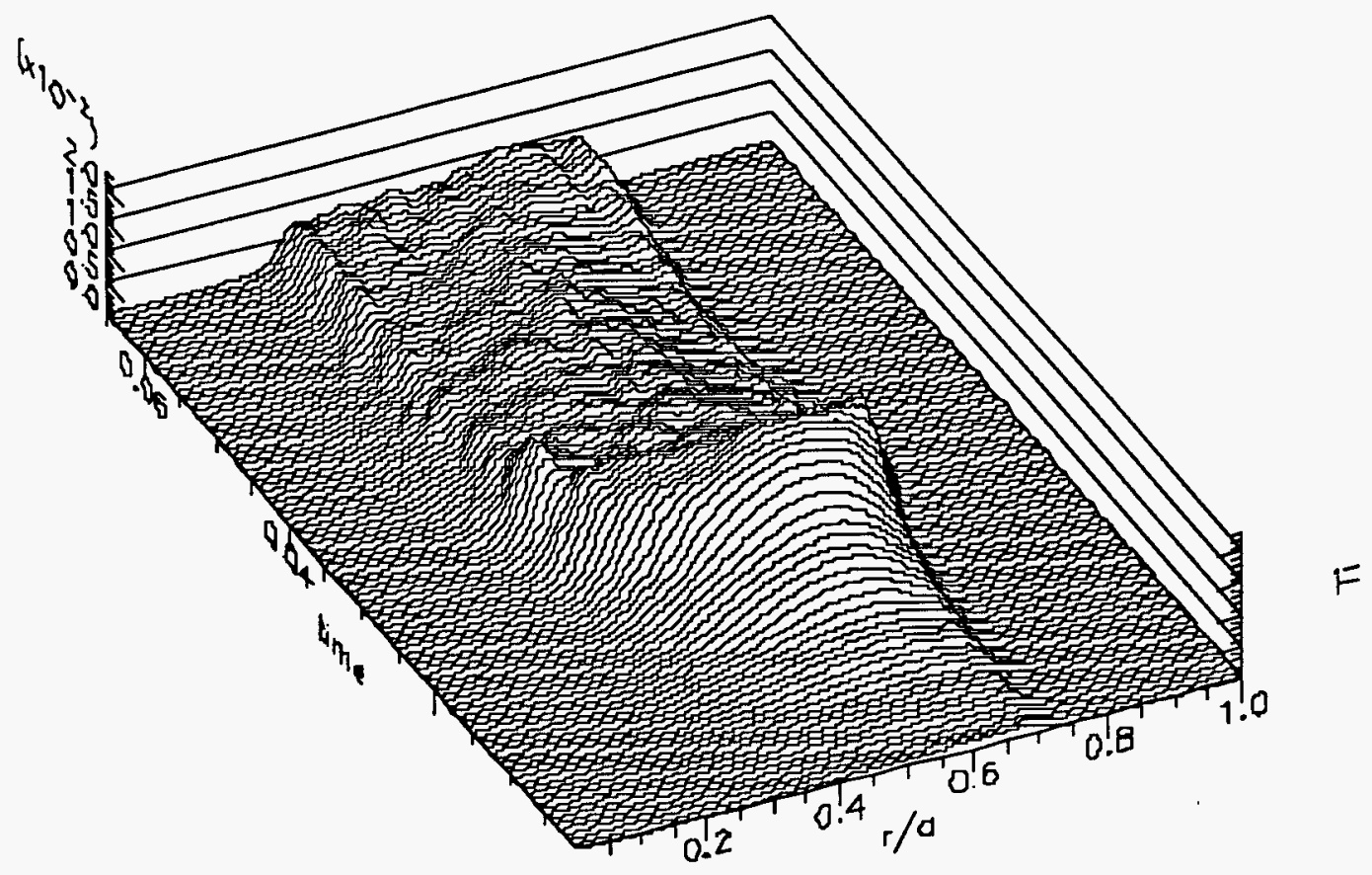

Fig. 13. The time history of the $T_{\mathrm{i}} \mathrm{rms}$ fluctuation profile.

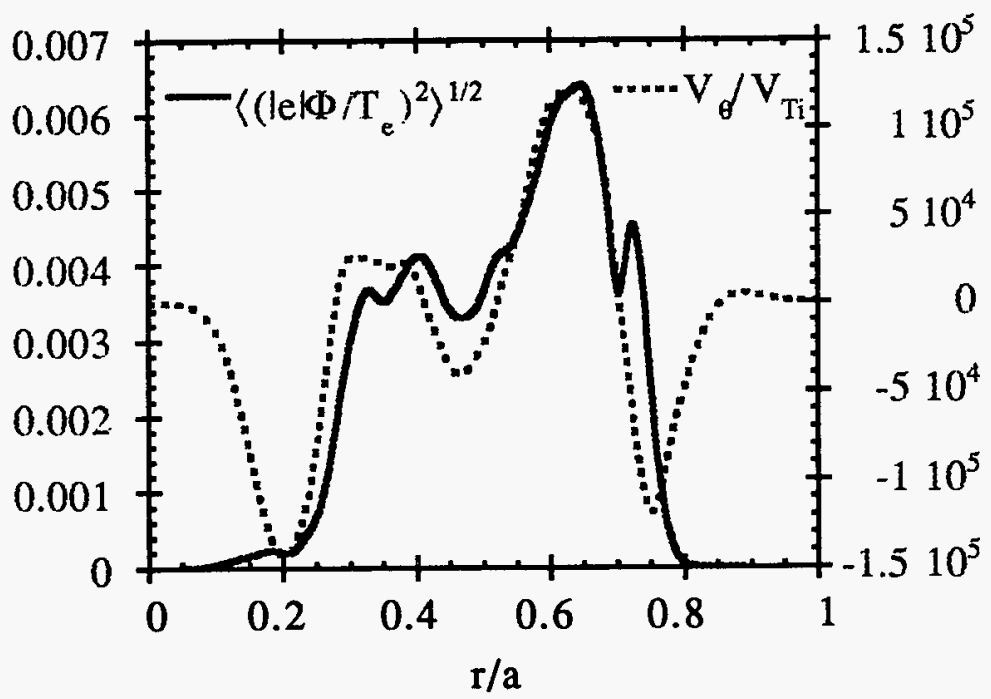

Fig. 14. The radial profiles of the electrostatic potential fluctuations and the poloidal flow. 


\section{Conclusions}

The parallel algorithm used for calculations of ion-temperature-gradient-driven turbulence has permitted successful completion of multi-helicity calculations on the T3E. Significant improvements have been realized by efficient packing of the communications messages before passing. For the present T3E and for the larger size calculations, 128 processors give the optimal performance. Computational issues that remain are:

1) The time spent in the convolution algorithm is the dominant part of the calculation. We have to develop a more efficient method for parallel calculation of the convolutions so as to minimize the loading and cache time.

2) Further refinements in the communications are needed to improve scalability.

The physics results are satisfactory and more calculations are needed to understand the effect of changing parameters.

The next step in this project is the development of full torus turbulence calculations with toroidal geometry. 


\section{References}

[1] J. W. Connor, M. Alexander, S. E. Attengerger, G. Bateman, D. Boucher, N. Chudnovskij, Y. N. Dnestrovskij, W. Dorland, A. Fukuyama, G. T. Hoang, D. Hogeweij, W. A. Houlberg, S. M. Kaye, J. E. Kinsey, J. A. Onings, M. Kotschenreuther, A. H. Kritz, V. M. Leonov, M. Marinucci, D. R. Ikkelsen, J. Ongena, A. R. Polevoi, F. Romanelli, D. Schissel, P. M. Stubberfield, T. Takizuka, A. Taroni, M. F. Turner, G. Vlad, R. E. Waltz, and J. Weiland, "Validation of 1-D transport and sawtooth models for ITER," in Sixteenth International Conference on Plasma Physics and Controlled Nuclear Fusion Research. Vienna: IAEA, 1996.

[2] B. Coppi, M. N. Rosenbluth, and R. Z. Sagdeev, "Instabilities due to temperature gradients in complex magnetic field configurations," Phys. Fluids, vol. 10, pp. 582-587, 1967.

[3] W. Horton, "Nonlinear Drift Waves and Transport in Magnetized Plasmas," Phys. Rep., vol. 192, pp. 1-177, 1990.

[4] G. S. Lee and P. H. Diamond, "Theory of Ion-Temperature-Gradient-Driven Turbulence in Tokamaks," Phys. Fluids, vol. 29, pp. 3291-3313, 1986.

[5] S. Hamaguchi and W. Horton, Phys. Fluids B, vol. 2, pp. 1833, 1990.

[6] H. Biglari and P. H. Diamond, "Theory of hydrodynamic trapped-ion-temperaturegradient-driven turbulence," Phys. Fluids B, vol. 3, pp. 1797-1800, 1991.

[7] G. W. Hammett and F. W. Perkins, "Fluid moment models for Landau damping with application to the ion-temperature-gradient instability," Phys. Rev. Lett., vol. 64, pp. 3019-3022, 1990.

[8] C. L. Hedrick and J. N. Leboeuf, "Landau fluid equations for electromagnetic and electrostatic fluctuations," Phys. Fluids B, vol. 4, pp. 3915-3934, 1992.

[9] R. E. Waltz, R. R. Dominguez, and G. W. Hammett, "Gyro-Landau fluid models for toroidal geometry," Phys. Fluids B, vol. 4, pp. 3138-3151, 1992.

[10] M. Kotschenreuther, W. Dorland, M. A. Beer, and G. W. Hammett, "Quantitative predictions of tokamak energy confinement from first-principles simulations with kinetic effects," Phys. Plasmas, vol. 2, pp. 2381-2389, 1995.

[11] W. Dorland, M. Kotschenreuther, M. A. Beer, G. W. Hammett, R. E. Waltz, R. R. Dominguez, P. M. Valanju, J. W. H. Miner, J. Q. Dong, W. Horton, F. L. Waelbroeck, T. Tajima, and M. J. LeBrun, "Comparisons of nonlinear toroidal turbulence simulations with experiment," in Proceedings of the 15th International Conference on Plasma Physics and Controlled Nuclear Fusion Research, vol. 3. Vienna: IAEA, 1994, pp. 463-474.

[12] L. Garcia, H. R. Hicks, B. A. Carreras, L. A. Charlton, and J. A. Holmes, "3D nonlinear MHD calculations using implicit and explicit time integrations schemes," Journal of Computational Physics, vol. 65, pp. 253-272, 1986.

[13] V. E. Lynch, B. A. Carreras, J. N. Leboeuf, B. C. Curtis, and R. L. Troutman, "Multi-CPU plasma fluid turbulence calculations on a Cray Y-MP C90," in Proceedings of Supercomputing '93, I. C. Society, Ed., 1993, pp. 308.

[14] V. E. Lynch, B. A. Carreras, J. B. Drake, J. N. Leboeuf, and P. Liewer, "Performance of a plasma fluid code on the Intel parallel computers," in Proceedings of Supercomputing '92, I. C. Society, Ed., 1992, pp. 286. 
|M97009358

(18) DOE, XF DCDDODE DOE

19971202057 